\title{
EFFECTIVENESS OF TWO-STAGE VERSUS ONE-STAGE INJECTION IN REDUCING DISCOMFORT DURING INFERIOR ALVEOLAR NERVE BLOCK IN CHILDREN
}

\author{
Randa Youssef Abd Al Gawad* and Nada Mohamed Wassef**
}

\begin{abstract}
Background: Inferior alveolar nerve block (IANB) anesthesia is a painful procedure \& many attempts are made to render the injection painless, especially in children. Aim: To assess discomfort expressed during modified two-stage IANB injection versus one-stage injection in 8-10 years old children.

Subjects \& Methods: Sixty children were randomly \& equally allocated to either intervention group (two-stage) or control group (one-stage). For all patients, topical anesthesia gel was applied. Intervention group: A short needle was used for the initial piercing \& deposition of the local anesthesia (LA), the needle was inserted for $4 \mathrm{~mm} \& 0.4 \mathrm{ml}$ of the solution was deposited, then withdrawn. After $5 \mathrm{~min}$, a long needle (gauge 27) was advanced till the point of LA drug deposition $\&$ bone touching \& the rest of the LA solution was deposited. Control group: A long needle was inserted, dropping $0.4 \mathrm{ml}$ of the solution incrementally till the point of LA drug deposition \& bone touching \& the rest of the LA solution was deposited. The overall patients' levels of discomfort were assessed using Face Legs Activity Cry Consolability scale (FLACC) \& Facial Image Scale (FIS). Results were tabulated \& statistically analyzed.
\end{abstract}

Results: For FLACC scale, the post-anesthetic values recorded a median=1 (range=0-3) in two-stages group, \& a median=2 (range=0-4) in one-stage group; with statistically significant difference between them $(\boldsymbol{p}=0.00)$. For FIS, a median=2 (range=1-4) in the two-stage group \& a median $=3$ (range=1-5) in one-stage group; the difference between groups was statistically significant $(\boldsymbol{p}=0.017)$.

Conclusion: The two-stage IANB injection technique is a simple \& an effective method of reducing the overall discomfort in children.

KEY WORDS: Discomfort, Inferior alveolar nerve block, Local anesthesia, one-stage IANB, two-stage IANB.

\footnotetext{
* Associate Prof. of Pediatric Dentistry and Dental Public Health, Faculty of Dentistry, Cairo University.

***ecturer of Pediatric Dentistry and Dental Public Health, Faculty of Dentistry, Cairo University.
} 


\section{INTRODUCTION}

Treating children with minimal discomfort \& pain has always been of paramount importance in pediatric dentistry \& continues to grow in necessity with the array of contemporary anesthetic techniques \& devices ${ }^{1}$. Even though local anesthetic (LA) injection is the most common method for pain control during dental procedures, it is the most anxiety-provoking factor for children ${ }^{2}$.

Pain is complex \& multidimensional, the control of which is crucial for establishing positive dental attitude in children ${ }^{3}$. Various techniques of reducing injection pain in children can be broadly categorized as psychological and physical. The psychological approach includes behavior management techniques, such as distraction \& hypnosis ${ }^{4}$. Physical means include various strategies, such as topical anesthesia application, warming injection solution, increased injection time, reducing the needle diameter, \& other recent techniques, such as WAND, electronic dental anesthesia, \& so forth. However, none of these techniques have been successful in eliminating fear $\&$ anxiety in children ${ }^{5}$.

Inferior alveolar nerve block (IANB) has been the most common technique used for anesthetizing mandibular teeth in elder children during both surgical and operative procedure. Even when this method successfully alleviates pain during dental treatment, the discomfort and anxiety produced during the administration of the anesthetic solution, as one-stage injection, become an obstacle for pediatric dentists and clinicians to establish a positive overall patient experience ${ }^{3-6}$

A two-stage injection has been suggested by some investigators in adults as a trial to control discomfort symptoms accompanied by the standard one-stage injection ${ }^{7}$. The first phase involves insertion of the needle submucosally, followed by deposition of $0.3-0.4 \mathrm{ml}$ anesthetic solution over 60 sec. After 4-5 min, the needle is reinserted gently to the proper depth and about $1-1.5 \mathrm{ml}$ of the solution is deposited (the second phase). This technique helps the clinician make a painless entry into the pre-anesthetized submucosa ${ }^{8,9}$.

While most of the literature describes the twostage IANB technique in adults as a practical alternative to the one-stage IANB, the evidence for its efficacy in children is lacking.

Therefore, the objective of this study is to compare between the discomfort due to two-stage and one-stage inferior alveolar nerve block injection techniques in children.

The hypothesis adopted in this study is a null hypothesis; that there is no difference in discomfort among children between two-stage and one-stage inferior alveolar nerve block injection techniques.

\section{SUBJECTS \& METHODS:}

This is a parallel single-blinded randomized clinical trial.

The study was initiated after obtaining Ethical Committee approval from Research Ethics Committee (REC), Faculty of Dentistry, Cairo University. Sample size of 60 children was estimated using $G$ Power 3.1.9.4 program according to the results of Rao et al., $2017^{10}$ (with $\alpha$ set at $0.05 \&$ power set at 0.8 ).

\section{Subjects}

Children were selected from the outpatient clinic, Pediatric Dentistry \& Dental Public Health Department, Faculty of Dentistry, Cairo University.

- Inclusion criteria:

- Children between 8 and 10 years of age.

- Patients indicated for IANB injection for their first time.

- Both genders are included.

○ Apparently healthy children.

○ Cooperative. 
- Exclusion criteria:

- Children who had taken CNS depressants or analgesics within 8 hours before the injection.

○ Children with acute dental pain.

- Children with mental or intellectual disabilities

Full detailed study protocol was explained to children and their guardians. Verbal assents were obtained from children \& signed informed consents were obtained. The patients were randomly allocated to either the intervention $(n=30)$ or control group $(n=30)$ using random.org program. The allocation sequence was concealed from the operator in sequentially numbered opaque sealed envelopes.

\section{Methods:}

Before anesthetic procedure, all children were assessed for discomfort assessment using Face Legs Activity Cry Consolability scale (FLACC) ${ }^{11}$, as an objective discomfort assessment scale (Table 1) \& Facial Image Scale (FIS) ${ }^{12}$, as a subjective discomfort assessment scale (Fig. 1) by the blinded calibrated investigator.

All anesthetic procedures were administered by the other investigator; who is well experienced $\&$ well trained in the two techniques. Routine behavior management pre-anesthetic protocol was adopted for all children. First the intraoral mucosal site was dried with a cotton pellet \& then flavored $20 \%$ benzocaine topical anesthesia gel (Iolite gel, Dharma Research Inc., USA) was applied for about $2 \mathrm{~min}$.

\section{Intervention group (modified two-stage IANB technique):}

The modified two-stage technique was administered as follows:

In stage one of the injections, a short needle (gauge 30) was used for the initial piercing \& deposition of the LA articaine drug Artinibsa 4\% with epinephrine 1:100,000 (Artinibsa, Inibsa,
Barcelona, Spain), the needle was inserted for $4 \mathrm{~mm}$ $\& 0.4 \mathrm{ml}$ of the solution was deposited slowly, then the needle was withdrawn.

In stage two, after $5 \mathrm{~min}$, landmark identification was made, a long needle (gauge 27) was advanced till the point of LA drug deposition \& bone touching. Aspiration to rule out any vascular entry was performed, and the rest of the anesthetic solution (1-1.2 mL) was deposited successively; where small amounts of LA solution deposited ahead of needle placement slowly over $1 \mathrm{~min}$ (successional technique, Multi-stage technique) ${ }^{10}$.

\section{Control group (standard one-stage IANB technique):}

A standard one-stage IANB injection ${ }^{8}$, where a long needle (gauge 27) was used for the initial piercing \& deposition of the LA drug after landmark identification. The needle was inserted \& dropping of $0.4 \mathrm{ml}$ of the solution incrementally \& slowly anesthetizing the pathway of the needle till the point of LA drug deposition \& bone touching. Similarly, aspiration $\&$ deposition of the rest of the anesthetic solution was done.

After finishing the anesthetic procedure, the patients' overall level of discomfort was assessed by the blinded calibrated investigator, using FLACC scale \& FIS. The results were then tabulated \& statistically analyzed.

Statistical analysis was performed using SPSS software program (SPSS 18; SPSS, Chicago, IL, USA). Data were explored for normality using Kolmogorov-Smirnov test of normality. Most values were non-parametric and were compared between groups using Mann Whitney U test, while pre-anesthesia and post-anesthesia values within the same group were compared using Wilcoxon-signed Rank test. For parametric data (age), Independent t test was used for 2 groups comparisons. The level of significance was set at $\boldsymbol{p}<0.05$. 
TABLE (1): Face Legs Activity Cry Consolability scale (FLACC) scale

\begin{tabular}{|c|c|c|c|}
\hline Criteria & Score 0 & Score 1 & Score 2 \\
\hline Face & $\begin{array}{c}\text { No particular expression or } \\
\text { smile }\end{array}$ & $\begin{array}{c}\text { Occasional grimace or frown, } \\
\text { withdrawn, uninterested }\end{array}$ & $\begin{array}{c}\text { Frequent to constant quivering } \\
\text { chin, clenched jaw }\end{array}$ \\
\hline Legs & Normal position or relaxed & Uneasy, restless, tense & Kicking, or legs drawn up \\
\hline Activity & $\begin{array}{c}\text { Lying quietly, normal position, } \\
\text { moves easily }\end{array}$ & $\begin{array}{c}\text { Squirming, shifting, back and } \\
\text { forth, tense }\end{array}$ & Arched, rigid or jerking \\
\hline Cry & No cry (awake or asleep) & $\begin{array}{c}\text { Moans or whimpers; } \\
\text { occasional complaint } \\
\text { Reassured by occasional } \\
\text { touching, hugging or being } \\
\text { talked to, distractible }\end{array}$ & $\begin{array}{c}\text { Crying steadily, screams or } \\
\text { sobs, frequent complaints }\end{array}$ \\
\hline
\end{tabular}

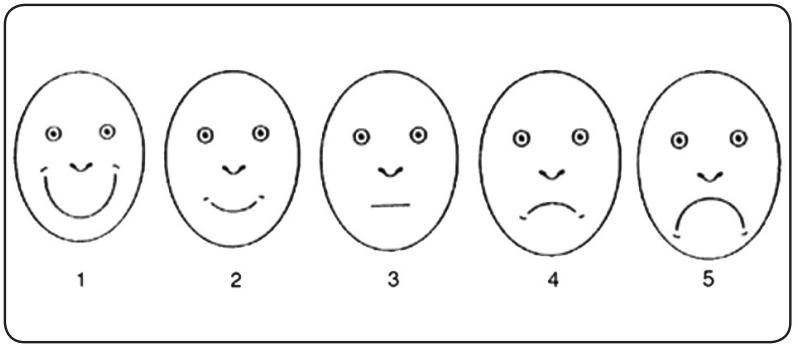

Fig (1): Facial image scale (FIS).

\section{RESULTS}

The mean age in two- stage group was $8.87 \pm 0.86$ years, while in one-stage group was $8.87 \pm 0.94$ years, with no statistically significant difference between groups $(\boldsymbol{p}=1)$.

\section{I-FLACC score:}

Comparison between both groups regarding preanesthetic \& post-anesthetic objective discomfort assessment and also within the same group are illustrated in Table (2).

\section{II-FIS score}

Comparison between both groups regarding preanesthetic \& post-anesthetic subjective discomfort assessment and also within the same group are illustrated in Table (3).

TABLE (2): Descriptive statistics, comparison of FLACC score between groups and FLACC scores before and after anesthesia injection within each group

\begin{tabular}{|c|c|c|c|c|}
\hline & Groups & $\begin{array}{c}\text { FLACC } \\
\text { (pre) }\end{array}$ & $\begin{array}{c}\text { FLACC } \\
\text { (post) }\end{array}$ & $\begin{array}{l}\mathrm{p}-\text { value } \\
\text { (pre-post) }\end{array}$ \\
\hline \multirow{3}{*}{$\begin{array}{l}\text { Intervention } \\
\text { (two- stage) }\end{array}$} & Median & 0.00 & 1.00 & \multirow{3}{*}{$0.00 *$} \\
\hline & Min & 0.00 & 0.00 & \\
\hline & Max & 2.00 & 3.00 & \\
\hline \multirow{3}{*}{$\begin{array}{c}\text { Control } \\
\text { (One-stage) }\end{array}$} & Median & 0.00 & 2.00 & \multirow{4}{*}{$0.059 \mathrm{~ns}$} \\
\hline & Min & 0.00 & 0.00 & \\
\hline & Max & 2.00 & 4.00 & \\
\hline \multicolumn{2}{|c|}{ p- value (between groups) } & $0.896 \mathrm{~ns}$ & $0.000^{*}$ & \\
\hline
\end{tabular}

Significance level $p<0.05$, *significant, $n s=$ non-significant 
TABLE (3): Descriptive statistics, comparison of FIS score between groups and FIS scores before and after anesthesia injection within the same group

\begin{tabular}{|c|c|c|c|c|}
\hline \multicolumn{2}{|c|}{ Groups } & \multirow{2}{*}{$\begin{array}{c}\text { FIS } \\
\text { pre }\end{array}$} & \multirow{2}{*}{$\begin{array}{l}\begin{array}{c}\text { FIS } \\
\text { post }\end{array} \\
2.00\end{array}$} & \multirow{2}{*}{$\begin{array}{c}\boldsymbol{p} \text { - value } \\
\text { (pre- } \\
\text { post) }\end{array}$} \\
\hline \multirow{3}{*}{$\begin{array}{l}\text { Intervention } \\
\text { (two- stage) }\end{array}$} & Median & & & \\
\hline & Min & 1.00 & 1.00 & \multirow[t]{2}{*}{$0.288 \mathrm{~ns}$} \\
\hline & Max & 4.00 & 4.00 & \\
\hline \multirow{3}{*}{$\begin{array}{c}\text { Control } \\
\text { (One -stage) }\end{array}$} & Median & 2.00 & 3.00 & \multirow{4}{*}{$0.789 \mathrm{~ns}$} \\
\hline & Min & 1.00 & 1.00 & \\
\hline & Max & 5.00 & 5.00 & \\
\hline \multicolumn{2}{|c|}{$p$ - value (between groups) } & $0.184 \mathrm{~ns}$ & $0.017 *$ & \\
\hline
\end{tabular}

Significance level $p<0.05$, *significant, ns=nonsignificant

\section{DISCUSSION}

Pain during IANB can be categorized as pain due to needle insertion, pain due to needle advacement, $\&$ solution deposition pain. Different techniques are employed to reduce this injection pain \& classified as follows: 1 . Techniques which reduce needle insertion pain as the application of topical anesthesia, 2. Techniques which minimize pain due to needle advacement as transcutaneous electrical nerve stimulation, 3. Techniques which decrease pain resulting from LA solution deposition as WAND, slow injection rate \& warming injection solution, \& finally 4 . Techniques which reduce the overall pain during IANB injection i.e. psychosocial interventions (distraction, hypnosis) ${ }^{13}$.

The two-stage IANB injection technique may present an alternative technique to the conventional one-stage inferior alveolar nerve block injection; where local analgesia of the tissues ahead of the needle can minimize insertion, placement, \& deposition pain resulting in the reduction of overall discomfort.

In the current study children aged 8-10 years old were enrolled in the study to ensure cooperation, cognitive \& intellectual development that ensure proper expression of their sensations \& feelings.

Apparently healthy chidren were enrolled in the study to exclude any medical, intellectual \&/ or psychological imbalances which may affect pain perception or FIS interpretation decision.

Patients indicated for IANB injection for their first time were selected \& a parallel design was adopted where a previous painful injection experience on one side (split-mouth technique) could lead to bias during the second injection due to the subjective fear factor setting into the child ${ }^{10}$.

Verbal assents were obtained from children beside the guardians' signed informed consents because their chronological age allowed this \& to ensure their cooperation during discomfort assessment.

Before anesthetic procedure, all children were assessed for discomfort status using FLACC as objective scale \& FIS as subjective one by the same calibrated investigator to ensure children's equality in discomfort expression. The pre-anesthetic values records revealed no statistically significant difference between the two groups. One blinded calibrated investigator recorded the pre \& postanesthetic FLACC \& FIS score values to avoid bias $\&$ ensure standardization.

FLACC scale was used as an objective behavioural pain assessment scale as it is simple, easy to be used \& suitable for children. The scale was first designed to be used in 2-7 years old children ${ }^{11}$, then it was used for older age groups ${ }^{10}$.

The FIS was used as a subjective discomfort record, since it is considered to be easy to be understood by children of this age group, sensitive $\&$ reproducable ${ }^{12}$. 
All anesthetic procedures were administered by the same operator for better standardization of the procedures.

The intraoral mucosal site was dried with a cotton pellet, then topical anesthesia gel was applied to aid in decreasing discomfort during needle insertion.

To rule out the bias of recording discomfort during needle insertion, needle advancement \& LA solution deposition reported in previous studies, specially in children, an overall discomfort assessment after completing anasethetic procedures, for the two groups, was adopted in this study.

Results of the present study show that there was a significant reduction of FLACC \& FIS scores when the two-stage technique was used. These results are previously \& strongly supported by Sandeep et al., $2016{ }^{13}$ who evaluated the practical efficacy of a twostage injection technique in reducing injection pain in 100 children (split-mouth, randomized controlled crossover trial) aged 7-13 using the Wong-Baker FACES Pain Rating Scale (FPS) \& Sound Eye Motor (SEM) scale.

Also, Rao et al., $2019^{10}$ compared the first stage of the modified two-stage technique during needle insertion \& LA solution deposition to the one-stage technique in 34 children aged 6-10 yrs (singleblinded comparative study) using the FLACC scale. Reaction of children in the first stage of the twostage group was significantly lower than that of the conventional one.

Reduction in discomfort may be due to the combination of the followings; in the two-stage IANB injection technique after the use of topical anasethesia, the initial needle penetration was only $4 \mathrm{~mm}$, thus decreasing discomfort during needle insertion. At the initial stage, the amount of solution deposition was only $0.4 \mathrm{ml}$ resulting in minimal tissue distension \& discomfort. During the waiting period $(5 \mathrm{~min})$, the optimal diffusion of LA drug can be expected to block the peripheral nerves thus, reducing needle placement \& deposition discomfort ${ }^{14}$.
The results also showed a statistically significant difference between the pre- and post-anesthetic FLACC scores in the one-stage group, denoting an increase in the level of discomfort experienced by the children.

Among the limitations of this study, that it was not possible for the assesssor recording the FLACC scale to be completely blinded, as one of the techniques involved insertion of the needle two times while the other involved only one insertion. However, in further studies this can be overcome by using a sham injection. Also other objective scales can be used as SEM (sound, eye, motor) scale that is simpler.

\section{CONCLUSIONS}

Based on the present findings, it can be concluded that IANB using the two-stage injection technique is a simple \& efficient technique minimizing the overall discomfort compared with the conventional one stage tecnique.

\section{REFERENCES}

1. Angelo $\mathrm{Z}$, Polyvios $\mathrm{C}$ Alternative practices of achieving anesthesia for dental procedures: a review J Dent Anesth Pain Med, 2018;18(2):79-88.

2. Milgrom P, Coldwell SE, Getz T, Weinstein P, RamsayDS. Four dimensions of fear of dental injections. J Am Dent Assoc, 1997; 128: 756-766.

3. Pinkham JR, Casamassimo PS, Fields HW, McTigue DJ, Nowak AJ. Pediatric Dentistry In: Infancy through Adolescence. 4th ed. St Louis, Mo. Elsevier Saunders 2005: 394-413.

4. ten Berge M, Veerkamp JS, Hoogstraten J, Prins PJ Childhood dental fear in the Netherlands: prevalence and normative data. Community Dent Oral Epidemiol, 2002;30: 101-107.

5. Oztas N, Ulusu T, Bodur H, Dogan C. The Wand in pulp therapy: an alternative to inferior alveolar nerve block. Quintessence Int, 2005;36: 559-564.

6. Ashwin R, Karthik S. A Modified Two Stage Technique of Administering an Inferior Alveolar Nerve Block in Children. J Nepal Dent Assoc, 2013;13:139-141. 
7. Nusstein J, Steinkruger G, Al Reader Beck M, Weaver J. The Effects of a 2-Stage Injection Technique on Inferior Alveolar Nerve Block Injection Pain. Anesth Prog, 2006;53:126-130.

8. Fayle SA, Duggal MS. In: Duggal MS, Curzon MEJ, Fayle SA, Toumba KJ, Robertson AJ, editors. Local analgesia. Restorative techniques in pediatric Dentistry. 2nd ed. London: Martin Dunitz; 2002: 13-27.

9. Walton RE, Reader A, Nusstein JM. In: Torabinejad M, Walton RE, Local Anesthesia. Endodontics Principles and Practice. 4th edition. St Louis, M: Saunders Elsevier; 2008: 129-147.

10. Rao A, Thakkar D, Rao A, Karuna YM, Srikant N Evaluation of a Modified Two-Stage Inferior Alveolar Nerve Block Technique: A Preliminary Investigation.
Dental Hypotheses, 2017; 8 (2):34-38.

11. Merkel S, Voepel-Lewis, T, Shayevitz J., Malviya, S. A behavioral scale for scoring postoperative pain in young children, by S Merkel and others, Pediatr Nurse, 1997 23(3): 293-297.

12. Fisk JD, Ritvo PG, Ross L, Haase DA, Marrie TJ, Schlech WF. Measuring the functional impact of fatigue: Initial validation of the Fatigue Impact Scale. Clin Infect Dis 1994a; 18(1): 79-83.

13. Sandeep V, Kumar, P M. Jyostna, D V. Evaluation of 2-Stage Injection Technique in Chilmdren Anesth Prog 2016; 63:3-7

14. Malamed SF. Handbook of Local Anesthesia. 5th ed. St Louis, Mo: C.V. Mosby; 2004:189-226. 